\title{
Patient Needs Should Drive Ventilator Selection for Stockpiling: "Handy" Devices May Not "Lend a Hand"
}

Following the attacks of September 11th, 2001, the people and government of the United States have been obsessed with disaster preparedness and threat levels. Travel has become more difficult, with metal detectors, explosive sniffing devices, and luggage searches. "Homeland security" has become a common phrase in Americans' lexicon. Recent natural events, from pandemic flu to earthquakes and tsunamis, have done little to calm our concerns.

Hospital preparedness has become a critical part of the disaster-management programs of cities and municipalities. This is colloquially referred to ${ }^{1}$ as "the space, the staff, and the stuff"- that is, the space where care will be given, which is most likely the hospital; the staff, or the available nurses, respiratory therapists, physicians, pharmacists, and other personnel trained to care for critically ill patients; and, finally, there is the stuff. For the respiratory therapist a major component of the stuff is the mechanical ventilator. Purchasing and stockpiling ventilators has dominated the ventilator sales landscape over the last several years. I estimate that more than 10,000 ventilators have been purchased for stockpiling around the United States, and that is a conservative estimate.

The Department of Homeland Security National Planning Guidelines coordinates and prioritizes emergency preparedness efforts at all response levels. The guidelines contain 15 National Planning Scenarios, at least two thirds of which involve mass-casualty respiratory failure. ${ }^{2}$ This is a critical piece of information. The mass-casualty respiratory failure scenario involves numerous patients with acute respiratory failure requiring intensive care, lung-protective ventilation, low tidal volume $\left(\mathrm{V}_{\mathrm{T}}\right)$, PEEP, sufficient flow capability, airway-pressure monitoring, and appropriate alarms. ${ }^{3-5}$ This type of scenario occurred most recently in the H1N1 pandemic, in which numerous patients had severe hypoxemic respiratory failure. ${ }^{6}$

Mechanical ventilators designed for mass-casualty care have been discussed in Respiratory CARe, and in a statement prepared by the American Association for Respiratory Care (AARC) to address the pertinent issues.$^{4,7}$ In this issue of the Journal, L'Her and Roy ${ }^{8}$ review 6 mass-casualty ventilators/resuscitators they describe as "handy." More specifically, they tested 4 portable volume controlled ventilators and 2 "automatic resuscitators," but the term "handy" applies in that the devices are small and light- weight, perhaps facilitating transport. L'Her and Roy also suggest that size and shape are important for stockpiling. This is an important issue that probably few institutions, except the Centers for Disease Control, have considered.

See the Original Study on Page 751

L'Her and Roy remind us that not all such devices are the same. ${ }^{8}$ Terminology plays an important role here. Two of the ventilators they studied are automatic resuscitators: the Vortran VAR-Plus and the Oxylator EMX. Food and Drug Administration approval of a resuscitator is a specific path-different and less stringent than for a ventilator. Resuscitators replace manual ventilation and, by definition, should not be used without direct one-on-one supervision. PEEP is a consequence of ventilator settings and lung mechanics. Compared to the resuscitators, the 4 volume ventilators L'Her and Roy tested were more consistent in $\mathrm{V}_{\mathrm{T}}$ delivery, more gas efficient, and were only nominally affected by changes in lung conditions. However, PEEP is available only with a disposable add-on device that may alter spontaneous breathing.

So, let's return to the "space." A change in the paradigm of patient care following a disaster suggests the hospital is where critical care will be provided. ${ }^{1}$ In this space we require a ventilator with sufficient capabilities to manage a patient with acute respiratory distress syndrome (ARDS), and these capabilities have been elaborated on previously..$^{2,4,5,7}$ So what about handy ventilators? There may be a place for their use outside the hospital, for ventilating patients who are on the way to definitive care, and in remote or temporary settings when access to definitive care is impeded by infrastructure collapse (Fig. 1).

What about stockpiling resuscitators? The Vortran VAR-1 was studied by Babic et al and found to deliver an unreliable $\mathrm{V}_{\mathrm{T}}$ and to be prone to failure. ${ }^{9} \mathrm{~L}$ 'Her and Roy describe this as "recurrent operational failures"; specifically, the device suddenly stopped ventilating the test lung, and then started again, and no explanation was found for the failure. This same failure was found by ECRI (formerly the Emergency Care Research Institute, but now known only by its acronym), which is an independent nonprofit health services research agency that performs comparative device evaluations and reviews (http:// 


\section{Patient Needs Should Drive Ventilator Selection for Stockpiling}

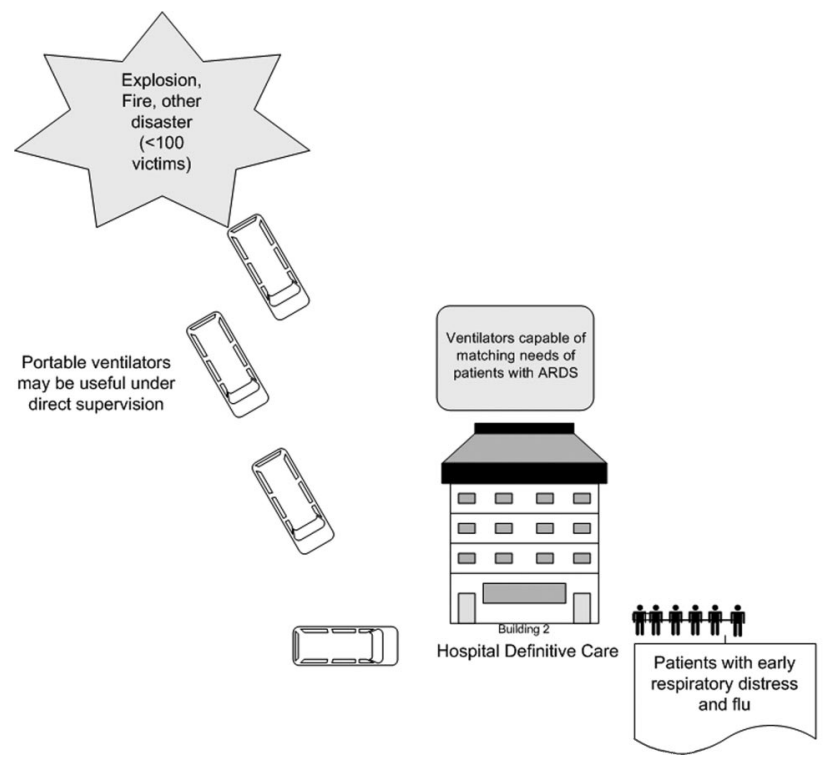

Fig. 1. Mass-casualty scenario, depicting the presentation of patients to the hospital. In an isolated disaster (eg, explosion, fire) the sickest patients are transferred to the hospital via ambulance, and during transport, under constant observation by emergency medical technicians, a portable ventilator/resuscitator may be useful. But in a situation such as an epidemic, where the patients are more likely to present to the hospital with evolving illness leading to acute respiratory distress syndrome (ARDS), we will need ventilators with adjustable tidal volume, PEEP, $\mathrm{F}_{\mathrm{IO}_{2}}$, and respiratory rate. In the hospital space these less functional devices have no role.

www.ecri.org). ECRI noted that the VAR "may spontaneously stop delivering breaths" without any alarm to alert of malfunction, and issued a hazard warning. ${ }^{10}$ Those findings have been questioned in the Journal, ${ }^{11}$ but the evidence against automatic resuscitators for use in mass-casualty respiratory failure continues to mount.

L'Her and Roy ${ }^{8}$ confirm the findings of Babic et al and ECRI. A resuscitator can replace a resuscitation bag in an emergency situation, though, unlike a person operating a resuscitation bag, the resuscitator has no feel for the $\mathrm{V}_{\mathrm{T}}$ or lung compliance. Mass-casualty respiratory failure would involve a large number of critically ill patients requiring the type of ventilator support outlined in the ARDS Network's $\mathrm{V}_{\mathrm{T}}$ study, ${ }^{12}$ and the staff will be over-tasked, so airway-pressure monitoring and appropriate audible and visual alarms will be essential. I have seen very little recognition of the fact that, the simpler a ventilator is, the fewer the number of alarms and monitors, and the less consistent the $\mathrm{V}_{\mathrm{T}}$ delivered, the more constant and expert attention is required. These shortcomings require that the clinician be more skilled, not less skilled. If the ventilator has no safety systems, clinical assessment is critical to safe and effective use. This is a conundrum that I believe has, so far, been lost on many decision makers.
Some particularly important observations and findings by L'Her and Roy are:

\footnotetext{
- The important difference between these disaster ventilators and standard emergency ventilators is the absence of disconnection alarms, which is, of course, a major patient-safety issue, but not less than a reliable delivered $\mathrm{V}_{\mathrm{T}}$ that is independent of the patient's characteristics.

- The pressure-cycled ventilators we tested, Oxylator EMX and VAR-Plus, should be ruled out because of the major impact of compliance and resistance on delivered $\mathrm{V}_{\mathrm{T}}$, and because of their high gas consumption. Our results suggest that Oxylator EMX and VAR-Plus would cause either hypoventilation (in a patient with low respiratory compliance) or severe barotrauma/volutrauma (in a patient with normal to high respiratory compliance).

- A contrario, an easy-to-use ventilator that cannot safely and effectively ventilate the patient is not a good choice. ${ }^{8}$
}

Portable handy ventilators probably could serve patients only at the scene of the disaster and/or on the way to the hospital, in the hands of a well trained individual. The simpler the ventilator/resuscitator, and the fewer alarms and monitoring, the better educated and trained the operator needs to be. A mass-casualty ventilator must be able to ventilate patients with hypoxemic respiratory failure, which requires adjustable PEEP, reliable low- $\mathrm{V}_{\mathrm{T}}$ delivery for lung protection, adjustable $\mathrm{F}_{\mathrm{IO}_{2}}$, monitoring of airway pressure, and alarms. None of the ventilators/resuscitators tested by L'Her and Roy have all those critical capabilities.

This is not to criticize the excellent, well conducted laboratory investigation by L'Her and Roy. ${ }^{8}$ They examined important characteristics of the tested devices, including ease of use and operational characteristics under different loads. The study is complete and provides data by which we can judge the devices. But a critical question remains unanswered. In the past 5 years the discussion of, research on, and planning for mass-casualty respiratory failure has moved from a poorly defined morass of several echelons of care to a more cogent plan. Treatment of masscasualty respiratory failure will predominantly take place in the hospital, where the staff and stuff are located. In that space, all the tools of critical care are available. We talk about ventilators, but what about syringes, airway equipment, drugs, intravenous pumps, suction catheters, and suction-generating devices? Those of us who work in intensive care units rarely worry about these critical accessories, because they are rarely in short supply.

To date, mechanical ventilation in the field is more likely in the case of catastrophic loss of infrastructure, 


\section{Patient Needs Should Drive Ventilator Selection for Stockpiling}

such as occurred in Haiti after the recent earthquake. If there is no space, the staff and the stuff must find their way to the patients. Under those conditions, stabilization with handy ventilators may be a stop-gap measure until definitive care can be brought to bear.

As H1N1 appeared de novo and taxed intensive care units around the world, there were no reported shortages of ventilators. Perhaps this should give us pause regarding the need for additional stockpiling and heighten our awareness about the unpredictability of viruses. So far the world has been fortunate that there have been no mass-casualty respiratory failure events big enough to tax the space, the staff, and the stuff. Planning for such an event requires evidence for determining the requirements for ventilators to be purchased. The AARC, the Centers for Disease Control, and the Task Force for Mass Critical Care have evaluated the needs and based the requirements of ventilators for stockpiling on these tenets. The pressure-cycled resuscitators (Oxylator EMX and VAR-Plus) tested by L'Her and Roy failed to meet the requirements for safe patient care. The volume-targeted ventilators (Carevent ALS, EPV100, Pneupac VR1, and Medumat Easy) had more consistent $\mathrm{V}_{\mathrm{T}}$ delivery, but do not have the adjustable PEEP, $\mathrm{V}_{\mathrm{T}}$, and respiratory rate to meet lung-protection standards.

Fear about mass-casualty events represents an opportunity for careful planning and an opportunity for commerce. Clearly the stockpiling of mass-casualty ventilators is part of sound planning, but the ventilator selection must not be based only on size and cost. An inexpensive device that cannot be safely used by the operator and cannot provide lung-protective ventilation is, in the end, just an inexpensive device with no clinical value. The requirements of the patients to be cared for must drive the selection of the ventilators stockpiled. More simply, automatic resuscitators should not be stockpiled where ventilators are needed. For short-term ventilation on the way to definitive care, such as in pre-hospital transport of patients, automatic resuscitators may prove useful.

Preparation for mass-casualty respiratory failure is a serious matter. It also represents serious dollars, which leads to serious competition between manufacturers. Planners are bombarded with evidence and testimonials from every manufacturer. Given the magnitude of this potential

\footnotetext{
Mr Branson has disclosed relationships with CareFusion, Covidien, Ikaria, Hamilton, and Advanced Circulatory Systems.

Correspondence: Richard D Branson MSc RRT FAARC, Department of Surgery, University of Cincinnati Medical Center, PO Box 670558, 231 Albert Sabin Way, Cincinnati OH 45267-0558. E-mail: richard.branson@ uc.edu.
}

DOI: $10.4187 /$ respcare. 01113 problem, the requirements advanced by the AARC, Centers for Disease Control, and Task Force on Mass Critical Care should be used to determine the characteristics of ventilators chosen. ${ }^{7,13}$

Over the past several years I have participated in several conferences on mass-casualty care, and I hope that masscasualty ventilators never need to be put into service. But the challenges of mass-casualty respiratory failure are real, and although we continue to prepare by day for the most likely eventualities, we might just as well pray that our preparation is never tested.

\section{Richard D Branson MSc RRT FAARC Department of Surgery University of Cincinnati Medical Center Cincinnati, Ohio}

\section{REFERENCES}

1. Rubinson L, O'Toole T. Critical care during epidemics. Crit Care 2005;9(4):311-313.

2. Department of Homeland Security. National preparedness guidelines. Washington, DC: DHS; September 2007. http://www.dhs.gov/ xlibrary/assets/National_Preparedness_Guidelines.pdf. Accessed April 12, 2011.

3. Rubinson L, Branson RD, Pesik N, Talmor D. Positive-pressure ventilation equipment for mass casualty respiratory failure. Biosecur Bioterror 2006;4(2):183-194.

4. Branson RD, Johannigman JA, Daugherty EL, Rubinson L. Surge capacity mechanical ventilation. Respir Care 2008;53(1):78-88; discussion 88-90.

5. Daugherty EL, Branson RD, Rubinson L. Mass casualty respiratory failure. Curr Opin Crit Care 2007;13(1):51-56.

6. Kumar A, Zarychanski R, Pinto R, Cook DJ, Marshall J, Lacroix J, et al. Critically ill patients with 2009 influenza A(H1N1) infection in Canada. JAMA 2009;302(17):1872-1879.

7. American Association for Respiratory Care. Guidelines for acquisition of ventilators to meet demands for pandemic flu and mass casualty incidents. Irving, TX: AARC; May 25, 2006. Updated January 30, 2008. http://www.aarc.org/resources/vent_guidelines_08.pdf. Accessed April 12, 2011.

8. L'Her E, Roy A. Bench tests of simple, handy ventilators for pandemics: performance, autonomy, and ergonomy. Respir Care 2011; 56(6):751-760.

9. Babic MD, Chatburn RL, Stoller JK. Laboratory evaluation of the Vortran automatic resuscitator model RTM. Respir Care 2007;52(12): 1718-1727.

10. Emergency Care Research Institute. Vortran VAR gas-powered resuscitators (also referred to as the Surevent) may spontaneously stop delivering breaths. Health Devices 2007;36(12):404-406.

11. Piper SD. Clarification of performance characteristics of the Vortran automatic resuscitator. Respir Care 2008;53(8):1089-1090.

12. National Institutes of Health Acute Respiratory Distress Syndrome Network. Ventilation with lower tidal volumes as compared with traditional tidal volumes for acute lung injury and the acute respiratory distress syndrome. N Engl J Med 2000;342(18):1301-1308.

13. Rubinson L, Hick JL, Curtis JR, Branson RD, Burns S, Christian MD, et al; Task Force for Mass Critical Care. Definitive care for the critically ill during a disaster: medical resources for surge capacity: from a Task Force for Mass Critical Care summit meeting, January 26-27, 2007, Chicago, IL. Chest. 2008;133(5 Suppl):32S-50S. 